\title{
DETERMINATION OF SPECTRA FROM PULSE-HEIGHT DATA*
}

\author{
ERIC B. ARNOLD
}

Randall Laboratory of Physics, The University of Michigan, Ann Arbor, Michigan 48104, U.S.A.

Received 3 February 1975

A method for determining parameters of a physical spectrum from pulse-height data is described. This method is designed to utilize information from the entire range of data and possesses advantages over other methods in use which arbitrarily restrict the input-data range used for parameter determination. Appli-

\section{Introduction}

In the analysis of pulse-height data or any similar data which can be related to some physical spectrum by a relation of the form:

$$
f(x)=\int_{\xi_{1}}^{\xi_{2}} \phi(\xi) R(x, \xi) \mathrm{d} \xi,
$$

where $f(x)$ is the measured (pulse-height) distribution, $\phi(x)$ is the physical spectrum, and $R(x, \xi)$ is the response function of the spectrometer; one usually has to determine $\phi(\xi)$ with $f(x)$ and $R(x, \xi)$ known. Various methods for obtaining solutions have been devised $^{1}$ ). The uniqueness of solutions to equations of this type depends strongly on the nature of the kernel or response function $\left.R(x, \xi)^{2}\right)$. In some cases an inverse $R^{-1}$ to $R$ exists such that a solution:

$$
\tilde{\phi}(\xi)=\int_{x_{1}}^{x_{2}} f(x) R^{-1}(\xi, x) \mathrm{d} x,
$$

may be obtained. However, $f(x)$ is generally known only approximately, and the resulting $\tilde{\phi}(\xi)$ may contain much noise, obscuring important behavior.

Often sufficient information is known about $\phi(\xi)$ that it may be written as a well-defined function of its independent variable $\xi$ and a set of undetermined parameters $\Lambda=\left\{\lambda_{1}, \ldots, \lambda_{k}\right\}$ :

$$
\phi(\xi)=\Theta\left(\xi ; \lambda_{1}, \ldots, \lambda_{k}\right)
$$

If this is so, a value for any $\lambda_{i}$ may be found by a fitting process, such as least-squares fitting, which seeks to find values for all elements of $A$ that are optimal in the sense of the fitting criterion. For example, if leastsquares fitting is used, one would seek values for

\footnotetext{
* Research supported in part by the National Science Foundation.
}

cations to two problems in $\beta$-spectroscopy are made. This method is most useful in those cases in which the information is distributed fairly smoothly over many data points. The statistical errors associated with the parameters may be determined in a straightforward manner.

$\lambda_{1}, \ldots, \lambda_{k}$ which would cause the expression:

$\chi^{2}=\int_{\xi_{1}}^{\xi_{2}} W(\xi)\left\{\tilde{\phi}(\xi)-\Theta\left(\xi, \lambda_{1}, \ldots, \lambda_{k}\right)\right\}^{2} \mathrm{~d} \xi$,

to be minimized, where $W(\xi)$ is a weighting function reflecting the statistical uncertainty of $\tilde{\phi}(\xi)$. While this approach has been used successfully in numerous cases, several things are likely to occur which can prevent it from providing useful results. One is that the uncertainties of the resulting parameters may be too large to make them meaningful. Another is that there may be several relative minima of $\chi^{2}$, and there may be no compelling reason to believe that one is really better than another, thus leaving the values of the $\lambda$ 's ambiguous. These things become more significant if $R^{-1}$ is uncertain, which it will be if $R$ is itself uncertain to some degree, which in practice is often the case. In fact it does not take much uncertainty in $R$ to make $R^{-1}$ completely meaningless, along with $\tilde{\phi}(\xi)$. Furthermore, there are many cases where $R^{-1}$ does not exist at all, whether or not $R$ is precisely defined, and this approach does nothing for them. It is therefore interesting to see what other approaches could take advantage of the information in eq. (3) without encountering the obstacles mentioned above.

\section{The present approach}

Rather than trying to reconstruct the physical spectrum through something like eq. (2), it is possible to attempt, at least formally, to reconstruct the measured distribution with a function of the form:

$f\left(x ; \lambda_{1}, \ldots, \lambda_{k}\right)=\int_{\xi_{1}}^{\xi_{2}} \Theta\left(\xi ; \lambda_{1}, \ldots, \lambda_{k}\right) R(x, \xi) \mathrm{d} \xi$.

Now a suitable fitting process may be used to choose values for the $\lambda$ 's in such a way that the fitting criterion 
is again satisfied in some optimal sense. For example, using a least-squares fitting process, and assuming that the measured distribution is obtained only at a discrete set $\left\{x_{j}\right\}$ of values of $x$, the $\lambda$ 's would be chosen in such a way that the expression:

$$
\chi^{2}=\sum_{j} \frac{\left\{f\left(x_{j}\right)-\tilde{f}\left(x_{j} ; \lambda_{1}, . ., \lambda_{k}\right\}^{2}\right.}{\sigma_{j}^{2}},
$$

is minimized, where $\sigma_{j}$ represents the uncertainty in the data and possibly in $\tilde{f}$ also, if the response function is somewhat uncertain. This process has an advantage of directness over that implied by eq. (4), and does not require special properties in the response function. Furthermore, it can handle uncertainties in the data and in the response function in a natural and straightforward fashion. While it is not the desired function $\phi(\xi)$ that is fitted, that function is determined in the process, and if it was not an appropriate function for the fitting job at hand, the resulting minimum value of $\chi^{2}$ will show this. In addition, by determining the variation of $\chi^{2}$ with each $\lambda_{i}$, the uncertainty of $\lambda_{i}$ may be determined; i.e. one can find out how much each parameter can change without spoiling the fit excessively, which gives a well-defined meaning to the uncertainty associated with $\lambda_{i}$.

\section{Examples of application}

This approach has been used in two different problems of $\beta$-spectroscopy. The differences between the problems serve to illustrate the considerable flexibility of this approach, since the problems differ significantly in the nature of the functions $\Theta\left(\xi ; \lambda_{1}, \ldots, \lambda_{k}\right)$ and $R(x, \xi)$. The same fitting criterion is used for both cases, which is a $\chi^{2}$ minimization. The actual fitting process is performed by a computer program derived from the program CURFIT described by Bevington ${ }^{3}$ ). It has been modified to include the option of fixing any of the parameters, with the effects of the uncertainties of these fixed values included in the calculations of $\chi^{2}$ and the uncertainties of the variable parameters. The usefulness of this modification will be seen in the first example. This fitting program is designed for fitting of functions which may have non-linear dependencies on the parameters. In order to carry out the fitting process, the partial derivatives of the fitting function with respect to the fitting parameters and some sort of reasonable initial estimate for the parameter values are required by the program in addition to the fitting function itself. In the examples presented below, it was generally not difficult to provide the needed initial estimates, and, if the fitting process converged at all, the final parameter values were fairly insensitive to the initial choices. The time required for convergence depended somewhat more on the initial parameter estimate, however, so that it was desirable to choose the initial estimate with some care. The parameters for which good estimates are most critical are those for which the fitting function is the most non-linear; parameters appearing linearly can have very rough initial estimates.

It should be noted that the fitted parameters do not have to be restricted to those which appear in the function $\Theta$. The response function could just as well depend on some or all of the same parameters as $\Theta$, as well as on some different ones. Then the fitting function (5) would depend on all of the parameters, even though some of them do not contribute to information about the physical spectrum. This feature will prove to be useful, again in the first example below. By this means some features of the response function may be determined at the same time as the spectrum. The fitting program is indifferent to the source of the parameters.

\subsection{Fitting PUlSe-height SPeCtra from a Si(Li) DETECTOR USED AT THE EXIT APERTURE OF A DOUBLE-FOCUSING MAGNETIC SPECTROMETER}

In this example the magnetic spectrometer provides the primary resolution, so that the energy resolution of the detector is used only as an aid to reduce background. The only parameter desired in this case is the total number of focused electrons. Since the detector pulse-height spectrum for monoenergetic electrons extends from a peak corresponding to total energy absorption down to zero, where it overlaps with detector and amplifier noise, one cannot simply count the total spectrum to obtain the desired number. Eliminating everything below a fixed level with a discriminator results in the inclusion of different fractions of the backscattering tail of the spectrum. Adjusting the discriminator level to correspond to changes in the magnetic field to keep this fraction constant would be tedious and likely to produce errors. It has been shown ${ }^{5}$ ) that, if the electron range is less than the depletion depth of the detector, the total-energyabsorption efficiency is essentially constant. Therefore a measure of the area of the total-energy-absorption peak should be proportional to the desired number. Thus the purpose of the analysis will be to extract this number from the pulse-height data with maximum accuracy and minimum arbitrariness.

On the basis of observations ${ }^{4,5}$ ) that the tail in the pulse-height spectrum due to backscattering is essen- 
tially constant, and with the assumption that the detector and amplifier noise is described by a Gaussian distribution, the response function for the detector is assumed to be of the following form:

$$
\begin{aligned}
R(v, E)= & \frac{\rho}{\sigma \sqrt{ }(2 \pi)} \exp \left[-(v-\eta E)^{2} / 2 \sigma^{2}\right]+ \\
& +\frac{1-\rho}{E \sigma \sqrt{ }(2 \pi)} \int_{E}^{\infty} \exp \left[-\left(v-\eta E^{\prime}\right)^{2} / 2 \sigma^{2}\right] \mathrm{d} E^{\prime},
\end{aligned}
$$

where $v$ is the pulse voltage, $E$ the energy of the incident electron, and $\rho, \sigma$, and $\eta$ are calibration parameters to be determined by the fitting program. A constant background is also assumed in the pulse-height spectrum. The source spectrum is assumed to be of the form:

$$
\Theta\left(E ; E_{\beta}\right)=N_{\beta} \delta\left(E-E_{\beta}\right)
$$

giving for the fitting function:

$$
\begin{aligned}
& \tilde{f}\left(v ; N_{\beta}, \rho, \sigma, \eta, N_{\mathrm{bkg}}\right)=\int_{E}^{\infty} \Theta\left(E ; E_{\beta}\right) R(v, E) \mathrm{d} E+N_{\mathrm{bkg}} \\
& =\int_{0}^{\infty} N_{\beta} \delta\left(E-E_{\beta}\right)\left\{\frac{\rho}{\sigma \sqrt{(2 \pi)}} \exp \left[-(v-\eta E)^{2} / 2 \sigma^{2}\right]+\right. \\
& \left.+\frac{1-\rho}{E \sigma \sqrt{(2 \pi)}} \int_{E}^{\infty} \exp \left[-\left(v-\eta E^{\prime}\right)^{2} / 2 \sigma^{2}\right] \mathrm{d} E^{\prime}\right\} \mathrm{d} E+N_{\mathrm{bkg}} \\
& =\frac{N_{\beta}}{\sigma \sqrt{(2 \pi)}}\left\{\exp \left[-\left(v-\eta E_{\beta}\right)^{2} / 2 \sigma^{2}\right]+\right. \\
& \left.+\frac{1-\rho}{E_{\beta}} \int_{E_{\beta}}^{\infty} \exp \left[-\left(v-\eta E^{\prime}\right)^{2} / 2 \sigma^{2}\right] \mathrm{d} E^{\prime}\right\}+N_{\mathrm{bkg}}
\end{aligned}
$$

$E_{\beta}$ is the energy of the focused electrons, which is determined by the magnetic field and therefore is fixed; $N_{\beta}$ is the desired parameter, namely, the number of focused electrons incident upon the detector. The remaining parameters are not related directly to the incident spectrum, although they could depend on the incident energy $E_{\beta}$. In the present case they are taken to be constant over the energy range of interest. This function has been found to represent the measured pulse-height spectrum very well in the vicinity of the total-energy-absorption peak.

Since a typical $\beta$-spectrometer run will consist of a series of measurements at different magnetic fields (and consequently different energies $E_{\beta}$ ), the parameters $\rho, \sigma, \eta$, and $N_{\text {bkg }}$ may be determined more precisely by averaging them over the whole set of spectra, taking into consideration whatever energy dependence might be expected for them. These values can then be regarded as fixed parameters, with known uncertainties, and the fitting of the pulse-height spectra can be repeated, this time fitting only $N_{\beta}$. It is also possible to perform this second fit over a restricted region, e.g. including only those channels which fall within, say, $\pm 3 \sigma$ of the center of the total-energy peak. Thus the fitting region can be chosen to include those channels for which the probability of detecting focused electrons is highest, providing the best background rejection. A gratifying result of this procedure has been to see that the fitted parameters do not depend significantly on the particular number of channels used in a fit; adding or subtracting one or more at either end of the fitting region does not cause a significant shift of fitted parameter values. Also gratifying was to find that the uncertainty for the number of counts in the peak was less than the square root of that number. This shows that, rather than losing accuracy in the process of subtracting off the tail underneath the peak, accuracy has been gained by deriving information from the shape of this tail.

\subsection{FitTING CONTINUOUS $\beta$-DECAY DATA FROM A MAGNETIC SPECTROMETER}

The physical spectrum in this example is assumed to be described by the Fermi theory of $\beta$-decay for allowed transitions, with provision for effects of a degenerate neutrino sea included. Again a constant background is assumed. This gives a physical spectrum which depends on four parameters: the effective source strength $N_{0}$, the endpoint energy of the $\beta$-spectrum $E_{0}$, the Fermi level for neutrinos $E_{\mathrm{f}}$, and the background level $N_{\mathrm{bkg}}$. At a magnetic field setting corresponding to electron momentum $p$, the number of electrons emitted by the source into the entrance aperture in momentum interval $\mathrm{d} p$ per unit time is:

$$
\begin{aligned}
& N\left(p ; N_{0}, E_{0}, E_{\mathrm{f}}\right) \\
& = \begin{cases}N_{0} p^{2}\left(E_{0}-E\right)^{2} F(Z, E), & 0 \leq E \leq E_{0}+E_{\mathrm{f}} ; \\
0, & E>E_{0}+E_{\mathrm{f}},\end{cases}
\end{aligned}
$$

where $E=\left[1+p^{2}\right]^{\frac{1}{2}}$ is the total electron energy in units with $m_{\mathrm{e}}=c=1$, and $F(Z, E)$ is the "Fermi function" which takes into account the effects of nuclear charge. The response function is derived from the property of magnetic $\beta$-spectrometers that the focusing is independent of the electron energy provided that the ratio of momentum to magnetic field remains constant. Consequently a carefully measured conversion-electron line may be scaled up or down in momentum to provide the response to a monoenergetic 
electron source of any desired momentum, assuming that energy losses in the source are negligibly different. Since in some cases they are not, it is desirable to use a conversion electron of energy close to the energy range under study, and to have the source mountings as nearly identical as possible. Unless a calibrated source is used or the transmission is known precisely from other considerations, the response function will only determine the relative probabilities of detecting an electron of momentum $p$ if the spectrometer field is given by $B$, but this is generally adequate; the constant of proportionality will be included in the parameter $N_{0}$ as determined by the fitting program. Since the response function used in this example was based on a measured electron line, it is initially defined only at discrete values of the magnetic field, and for a particular scale change it may not be defined at the value of the magnetic field for which it is needed, so an interpolation scheme is required. The measured line is first smoothed by representing it as a Fourier series over the region where it is non-zero, and then truncating the series at a point where $\chi^{2} / n$ no longer decreases with increasing $n$, where $n$ is the number of Fourier series terms used, and $\chi^{2}$ is evaluated for the truncated series. The original values are then replaced by the values of the truncated series at the same points, and then fourpoint interpolation is used to evaluate the response function between the original points (the Fourier series itself could have been used for this purpose, but it is more time-consuming and no more meaningful). This is the function $R(B, p)$ used below. It is non-zero only in a region:

$$
\rho_{\min }<p / B<\rho_{\max },
$$

or:

$$
p_{\min }<p<p_{\max },
$$

where $p_{\min (\max )}=B \rho_{\min (\max )}$. Thus the fitting function to be used in this case looks like:

$$
\begin{aligned}
& \tilde{N}\left(B ; N_{0}, E_{0}, E_{\mathrm{f}}, N_{\text {bkg }}\right) \\
& =\int_{p_{\min }}^{p_{\max }}\left\{N\left(p ; N_{0}, E_{0}, E_{\mathrm{f}}\right)+N_{\text {bkg }}\right\} R(B, p) \mathrm{d} p .
\end{aligned}
$$

Recalling the expression (10) for $N\left(p ; N_{0}, E_{0}, E_{\mathrm{f}}\right)$ and the nature of $R(B, p)$ a numerical integration is called for in the evaluation of this fitting function. While some time might have been saved by using the same values of the integration variable in this process for which $R(B, p)$ was directly defined, it turns out to be convenient to have $R(B, p)$ defined over the continuous interval $\left[p_{\min }, p_{\max }\right]$ for other reasons which will appear below. Since this analysis is intended to be as sensitive as possible to small details near the endpoint of the spectrum, and since the shape of the spectrum in the vicinity of the endpoint is essentially quadratic, it is reasonable to use an integration formula which represents areas under quadratic curves exactly, providing for less error in the near vicinity of the endpoint than would be obtained by using trapezoidal or mean-value methods, which would be as reasonable as any method based on equally spaced subintervals such as would be provided by the original response-function data points. Thus the usefulness of having the interpolation scheme for the response function comes in being able to choose the points at which the integrand is evaluated in any desired fashion. Since 100 data points were measured to define the response function, it would be inappropriate to use more points in the numerical integration, and therefore 50 subintervals containing two points each were used. The points were chosen in each subinterval according to the Gaussian two-point integration method, which gives areas of curves up to the third degree exactly. The response function has to be evaluated at these points only once, since the same values are used on each repeat of the numerical integration and can be stored for repeated use. The fitting procedure requires the derivatives of the fitting function (11) with respect to the fitted parameters, which are $N_{0}, E_{0}, E_{\mathrm{f}}$, and $N_{\mathrm{bkg}}$. For $N_{0}, E_{0}$, and $N_{\text {bkg }}$ the simplest way to obtain the derivatives is to differentiate the sum which replaces (11) since (11) is evaluated numerically. This results in more sums, most parts of which are already available from the numerical integration, so it is not much additional work to calculate these derivatives at the same time the fitting function is calculated.

The dependence on $E_{\mathrm{f}}$ is sufficiently different to make a special note. As long as the range of integration $\left[p_{\min }, p_{\max }\right]$ corresponds to values of electron energy less than $E_{0}+E_{\mathrm{f}}$, the fitting function does not depend on $E_{\mathrm{f}}$. However, when $E_{0}+E_{\mathrm{f}}$ falls between the energies corresponding to $p_{\min }$ and $p_{\max }$, the upper limit is replaced by:

$$
p_{\mathrm{f}}=\left[\left(E_{0}+E_{\mathrm{f}}\right)^{2}-1\right]^{\frac{1}{2}}
$$

The evaluation of the fitting function will now have fewer than 50 complete subintervals, with a smaller interval from the end of the highest regular interval under $p_{\mathrm{f}}$ to $p_{\mathrm{f}}$. The response function must be evaluated at two points in this smaller interval along with the rest of the integrand, since the previously calculated values of the response function are valid only for the standard interval. Thus the ability to interpolate the 
response function is necessary for this last subinterval. The derivative of the fitting function with respect to $E_{\mathrm{f}}$ is much more easily calculated from (11) using $p_{\mathrm{f}}$ as the upper limit than from the sum resulting from the numerical integration.

Thus the requirements of the fitting program are met by this function. A curious feature requiring some careful attention is that the number of parameters fitted varies over the spectrum, depending on whether $p_{\mathrm{f}}$ falls in the interval $\left[p_{\min }, p_{\text {max }}\right]$ or not. However, this is not a fundamental difficulty; it must be considered appropriately when statistical tests involving the number of degrees of freedom are made.

\section{Concluding remarks}

It has been shown that a fitting function to represent measured data may be obtained if the physical spectrum can be given by a definite functional form with undetermined parameters, and if the response of the spectrometer to a $\delta$-function input is known over the range of the input spectrum. The response may be measured experimentally and contain experimental error. A significant feature of this method is that it uses all of the data in the determination of the unknown parameters. This should prove to be advantageous over other methods ${ }^{6}$ ) which use only part of the data for the determination of certain parameters. While a sharp peak or step lends itself rather well to this latter analysis, cases lacking such distinctive features are better treated in the manner described in this work. In general, this method should be most appropriate in cases wherein the information sought is distributed rather smoothly over many adjacent data points. A particular example is found whenever details smaller than the line width of the spectrometer are to be investigated. Two distinct advantages of this method are the lack of arbitrariness in choosing a region of data for the determination of specific parameters, and the ease with which the uncertainties associated with the resulting parameter values are determined.

It is clear that determining the response of the spectrometer is necessary for this method to be useful, and that may be a difficult task. However, even a rather crude representation of the spectrometer response can be utilized with the expectation of better results than would be obtained without considering spectrometer responses. In difficult cases, Monte-Carlo methods could provide a very practical approach to the problem of determining spectrometer response.

The author wishes to express his appreciation for the continued support of Dr M. L. Wiedenbeck and the helpful comments of Drs J. C. Zorn and B. P. Roe.

\section{References}

1) See, for example: D. W. Green, Nucl. Instr. and Meth. 76 (1969) 349 ; V. R. Saulit, Izv. Akad. Nauk. SSSR, Ser. Fiz. 37, no. 8 (1973) 1671 (Engl. transl.); see also Bergkvist (ref. 6).

2) F. G. Tricomi, Integral equations (Interscience, New York, 1957) p. 5.

3) P. R. Bevington, Data reduction and error analysis for the physical sciences (McGraw-Hill, New York, 1969) p. $235 \mathrm{ff}$.

4) M. J. Berger et al., Nucl. Instr. and Meth. 69 (1969) 181.

$\left.{ }^{5}\right)$ M. Waldschmidt and S. Wittig, Nucl. Instr. and Meth. 64 (1968) 159.

6) For example, see: K. E. Bergkvist, Nucl. Phys. B37 (1972) 317, especially pp. $354 \mathrm{ff}$; see also: J. Law, Phys. Rev. D7, 11 (1973) 3314 